\title{
Vorwort Heft 3-10
}

\author{
Hans-Christoph Grunau
}

Online publiziert: 10 . August 2010

(C) Vieweg+Teubner und Deutsche Mathematiker-Vereinigung 2010

Das Herausgebergremium hatte sich überlegt, dass es grundsätzlich wünschenswert sei, „Themenhefte“ zu gestalten. Denn auf diese Weise könne eine Thematik aus verschiedenen Perspektiven beleuchtet werden und es ergebe sich ein unmittelbarerer Eindruck von deren Vielseitigkeit und Vielschichtigkeit.

In diesem - und aller Voraussicht nach auch im nächsten - Heft ist der Jahresbericht nun in der glücklichen Situation, dass die druckfertig vorliegenden Manuskripte die Gestaltung eines derartigen ,Themenheftes“ gestatten. Im vorliegenden Heft finden Sie zwei Übersichtsartikel zu dem Themenkomplex „Partielle Differentialgleichungen“, der von dem einen Beitrag aus der Perspektive ,geometrische Eigenschaften von Lösungen“ und von dem anderen Beitrag aus der Perspektive „Regularität und Abschätzungen von Lösungen“ betrachtet wird.

Stellen Sie sich z.B. die möglichen Eigenschwingungen einer eingespannten kreisförmigen Membran ( $\rightarrow$ Pauke) vor. Hier kann man das entsprechende Eigenwertproblem (für das Dirichletproblem der Laplacegleichung) explizit analysieren: Das räumliche Profil der Grundschwingung ist positiv und radialsymmetrisch, während man bei der ersten Oberschwingung zwei Bereiche entgegengesetzten Vorzeichens und lediglich noch Axialsymmetrie beobachtet. Die weiteren Oberschwingungen werden nun immer komplizierter und weisen sukzessive ein immer geringeres Maß an Symmetrie auf. Tobias Weth stellt diese experimentellen Beobachtungen in seinem Übersichtsartikel ,Symmetry of solutions to variational problems for nonlinear elliptic equations via reflection methods" in den Kontext einer allgemeinen und gleichermaßen relativ elementaren Theorie in dem Sinne, dass er sich auf Reflektions-

H.-Ch. Grunau ( $\bowtie)$

Institut für Analysis und Numerik, Fakultät für Mathematik, Otto-von-Guericke-Universität, Postfach 4120, 39016 Magdeburg, Deutschland

e-mail: hans-christoph.grunau@ovgu.de 
methoden beschränkt und lediglich auf wohletablierte analytische und topologische Werkzeuge verweisen muss.

Um Existenz von Lösungen für Randwertprobleme bei partiellen Differentialgleichungen zu zeigen, besteht ein typischer Zugang darin, zunächst ,a prioriAbschätzungen" für als existent angenommene Lösungen herzuleiten. Sind diese Abschätzungen hinreichend gut, so ergeben funktionalanalytische Hilfsmittel auch sofort Existenz. Bei linearen ,elliptischen“ Gleichungen (Prototyp ist die Laplacegleichung $-\Delta u=f$ ) heißen die grundlegenden Abschätzungen in Räumen klassisch differenzierbarer Funktionen nach Schauder und in Räumen schwach differenzierbarer Funktionen mit $L^{\gamma}$-Integralnormen nach Calderón und Zygmund. Giuseppe Mingione gibt nun einen bemerkenswerten Überblick über „Nonlinear aspects of CalderónZygmund theory". Er betrachtet eine allgemeine Klasse nichtlinearer elliptischer Differentialgleichungen und stellt im $L^{\gamma}$-Kontext mittels neuer methodischer Zugänge zahlreiche aktuelle Regularitäts- und Abschätzungsaussagen zusammen, wie man sie vorher oft nur von linearen Problemen her kannte. Zum Einstieg in diese Thematik lässt er die klassischen Tatsachen aus der linearen Theorie zunächst Revue passieren.

Einen Kontrapunkt zu soviel partiellen Differentialgleichungen in diesem Heft bilden die Buchbesprechungen. Hier werden neue Monographien unter anderem aus der Stochastik, der Finanzmathematik und der symplektischen Geometrie vorgestellt. Zwar sind auch hier die partiellen Differentialgleichungen mit einem Titel präsent, der allerdings hyperbolische Gleichungen und diese zudem in zufälligen Medien zum Gegenstand hat. 